\title{
Derivations, local and 2-local derivations on some algebras of operators on Hilbert $\mathrm{C}^{*}$-modules
}

\author{
Jun He, Jiankui Li, and Danjun Zhao \\ Department of Mathematics, East China University of Science and Technology \\ Shanghai 200237, China
}

\begin{abstract}
For a commutative $\mathrm{C}^{*}$-algebra $\mathcal{A}$ with unit $e$ and a Hilbert $\mathcal{A}$-module $\mathcal{M}$, denote by $\operatorname{End}_{\mathcal{A}}(\mathcal{M})$ the algebra of all bounded $\mathcal{A}$-linear mappings on $\mathcal{M}$, and by $\operatorname{End}_{\mathcal{A}}^{*}(\mathcal{M})$ the algebra of all adjointable mappings on $\mathcal{M}$. We prove that if $\mathcal{M}$ is full, then each derivation on $\operatorname{End}_{\mathcal{A}}(\mathcal{M})$ is $\mathcal{A}$-linear, continuous, and inner, and each 2-local derivation on $\operatorname{End}_{\mathcal{A}}(\mathcal{M})$ or $\operatorname{End}_{\mathcal{A}}^{*}(\mathcal{M})$ is a derivation. If there exist $x_{0}$ in $\mathcal{M}$ and $f_{0}$ in $\mathcal{M}^{\prime}$, such that $f_{0}\left(x_{0}\right)=e$, where $\mathcal{M}^{\prime}$ denotes the set of all bounded $\mathcal{A}$-linear mappings from $\mathcal{M}$ to $\mathcal{A}$, then each $\mathcal{A}$-linear local derivation on $\operatorname{End}_{\mathcal{A}}(\mathcal{M})$ is a derivation.
\end{abstract}

Keywords: Derivations, Hilbert $\mathrm{C}^{*}$-modules, inner derivations, local derivations, 2-local derivations

Mathematics Subject Classification(2010): 47B47; 47L10

\section{Introduction and preliminaries}

The structure of derivations on operator algebras is an important part of the theory of operator algebras.

Let $\mathcal{A}$ be an algebra and $\mathcal{M}$ be an $\mathcal{A}$-bimodule. Recall that a derivation is a linear mapping $d$ from $\mathcal{A}$ into $\mathcal{M}$ such that $d(x y)=d(x) y+x d(y)$, for all $x, y$ in $\mathcal{A}$. For each $m$ in $\mathcal{M}$, one can define a derivation $D_{m}$ by $D_{m}(x)=m x-x m$, for all $x$ in $\mathcal{A}$. Such derivations are called inner derivations.

It is a classical problem to identify those algebras on which all derivations are inner derivations. Several authors investigate this topic. The following two results are

\footnotetext{
${ }^{*}$ Corresponding author. E-mail address: jiankuili@yahoo.com
} 
classical. S. Sakai [17] proves that all derivations from a $\mathrm{W}^{*}$-algebra into itself are inner derivations. E. Christensen [3] proves that all derivations from a nest algebra into itself are inner derivations.

In 1990, R. Kadison [10] and D. Larson, A. Sourour [13] independently introduce the concept of local derivation in the following sense: a linear mapping $\delta$ from $\mathcal{A}$ into $\mathcal{M}$ such that for every $a \in \mathcal{A}$, there exists a derivation $d_{a}: \mathcal{A} \rightarrow \mathcal{M}$, depending on $a$, satisfying $\delta(a)=d_{a}(a)$. In [10], R. Kadison proves that each continuous local derivation from a von Neumann algebra into its dual Banach module is a derivation. In [13, D. Larson and A. Sourour prove that each local derivation from $B(\mathcal{X})$ into itself is a derivation, where $\mathcal{X}$ is a Banach space. B. Jonson [8] proves that each local derivation from a $\mathrm{C}^{*}$-algebra into its Banach bimodule is a derivation. Z. Pan and the second author of this paper [14] prove that each local derivation from the algebra $\mathcal{M} \cap \operatorname{alg} \mathcal{L}$ into $B(\mathcal{H})$ is a derivation, where $\mathcal{H}$ is a Hilbert space, $\mathcal{M}$ is a von Neumann algebra acting on $\mathcal{H}$, and $\mathcal{L}$ is a commutative subspace lattice in $\mathcal{M}$. For more information about this topic, we refer to [2, 4, 6].

In 1997, P. Šemrl [18] introduces the concept of 2-local derivations. Recall that a mapping $\delta: \mathcal{A} \rightarrow \mathcal{M}$ (not necessarily linear) is called a 2-local derivation if for each $a, b \in \mathcal{A}$, there exists a derivation $d_{a, b}: \mathcal{A} \rightarrow \mathcal{M}$ such that $\delta(a)=d_{a, b}(a)$ and $\delta(b)=d_{a, b}(b)$. Moreover, the author proves that every 2-local derivation on $B(\mathcal{H})$ is a derivation for a separable Hilbert space $\mathcal{H}$. J. Zhang and H. Li [19] extend the above result for arbitrary symmetric digraph matrix algebras and construct an example of 2local derivation which is not a derivation on the algebra of all upper triangular complex $2 \times 2$ matrices. S. Ayupov and K. Kudaybergenov [1] prove that each 2-local derivation on a von Neumann algebra is a derivation. For more information about this topic, we refer to [2, 7, 11].

In this paper, we study derivations, local derivations and 2-local derivations on some algebras of operators on Hilbert $\mathrm{C}^{*}$-modules. There are few results in this topic. P. Li, D. Han and W. Tang [15] prove that each derivation on $\operatorname{End}_{\mathcal{A}}^{*}(\mathcal{M})$ is inner, where $\mathcal{M}$ is a full Hilbert $\mathrm{C}^{*}$-module over a commutative unital $\mathrm{C}^{*}$-algebra $\mathcal{A}$. M. Moghadam, M. Miri and A. Janfada [16] prove that each $\mathcal{A}$-linear derivation on $\operatorname{End}_{\mathcal{A}}(\mathcal{M})$ is inner, where $\mathcal{M}$ is a full Hilbert $\mathrm{C}^{*}$-module over a commutative unital $\mathrm{C}^{*}$-algebra $\mathcal{A}$ with the property that there exist $x_{0}$ in $\mathcal{M}$ and $f_{0}$ in $\mathcal{M}^{\prime}$ such that $f_{0}\left(x_{0}\right)=e$.

Hilbert $\mathrm{C}^{*}$-modules provide a natural generalization of Hilbert spaces by replacing the complex field $\mathbb{C}$ with an arbitrary $\mathrm{C}^{*}$-algebra. The theory of Hilbert $\mathrm{C}^{*}$-modules plays an important role in the theory of operator algebras, as it can be applied in many fields, such as index theory of elliptic operators, K- and K K-theory, noncommutative geometry, and so on.

In the following, we would firstly review some properties of Hilbert $\mathrm{C}^{*}$-modules [12]. Let $\mathcal{A}$ be a $\mathrm{C}^{*}$-algebra and $\mathcal{M}$ be a left $\mathcal{A}$-module. 
$\mathcal{M}$ is called a Pre-Hilbert $\mathcal{A}$-module if there exists a mapping $\langle\cdot, \cdot\rangle: \mathcal{M} \times \mathcal{M} \longrightarrow \mathcal{A}$ with the following properties: for each $\lambda \in \mathbb{C}, a \in \mathcal{A}, x, y, z \in \mathcal{M}$,

(1) $\langle x, x\rangle \geq 0$, and $\langle x, x\rangle=0$ implies that $x=0$,

(2) $\langle\lambda x+y, z\rangle=\lambda\langle x, z\rangle+\langle y, z\rangle$,

(3) $\langle a x, y\rangle=a\langle x, y\rangle$,

(4) $\langle x, y\rangle=\langle y, x\rangle^{*}$.

The mapping $\langle\cdot, \cdot\rangle$ is called an $\mathcal{A}$-valued inner product. The inner product induces a norm on $\mathcal{M}:\|x\|=\|\langle x, x\rangle\|^{1 / 2}$. $\mathcal{M}$ is called a Hilbert $\mathcal{A}$-module (or more exactly, a Hilbert $\mathrm{C}^{*}$-module over $\mathcal{A}$ ), if it is complete with respect to this norm.

We denote by $\langle\mathcal{M}, \mathcal{M}\rangle$ the closure of the linear span of all the elements of the form $\langle x, y\rangle, x, y \in \mathcal{M} . \mathcal{M}$ is called a full Hilbert $\mathcal{A}$-module if $\langle\mathcal{M}, \mathcal{M}\rangle=\mathcal{A}$.

For a full Hilbert $\mathcal{A}$-module $\mathcal{M}$, we have the following lemma.

Lemma 1.1. Let $\mathcal{A}$ be a $C^{*}$-algebra with unit $e$ and $\mathcal{M}$ be a full Hilbert $\mathcal{A}$-module. There exists a sequence $\left\{x_{i}\right\}_{i=1}^{n} \subseteq \mathcal{M}$, such that $\sum_{i=1}^{n}\left\langle x_{i}, x_{i}\right\rangle=e$.

A linear mapping $T$ from $\mathcal{M}$ into itself is said to be $\mathcal{A}$-linear if $T(a x)=a T(x)$ for each $a \in \mathcal{A}$ and $x \in \mathcal{M}$. A bounded $\mathcal{A}$-linear mapping from $\mathcal{M}$ into itself is called an operator on $\mathcal{M}$. Denote by $\operatorname{End}_{\mathcal{A}}(\mathcal{M})$ all operators on $\mathcal{M}$. $\operatorname{End}_{\mathcal{A}}(\mathcal{M})$ is a Banach algebra.

A mapping $T$ from $\mathcal{M}$ into itself is said to be adjointable if there exists a mapping $T^{*}$ such that $\langle T x, y\rangle=\left\langle x, T^{*} y\right\rangle$, for all $x, y \in \mathcal{M}$. Notice that each adjointable mapping must be an operator. Denote by $\operatorname{End}_{\mathcal{A}}^{*}(\mathcal{M})$ all adjointable operators on $\mathcal{M}$. $\operatorname{End}_{\mathcal{A}}^{*}(\mathcal{M})$ is a $\mathrm{C}^{*}$-algebra.

Similarly, a linear mapping $f$ from $\mathcal{M}$ into $\mathcal{A}$ is said to be $\mathcal{A}$-linear if $f(a x)=a f(x)$ for each $a \in \mathcal{A}$ and $x \in \mathcal{M}$. The set of all bounded $\mathcal{A}$-linear mappings from $\mathcal{M}$ to $\mathcal{A}$ is denoted by $\mathcal{M}^{\prime}$.

For each $x$ in $\mathcal{M}$, one can define a mapping $\hat{x}$ from $\mathcal{M}$ to $\mathcal{A}$ by follows: $\hat{x}(y)=\langle y, x\rangle$, for all $y \in \mathcal{M}$. Obviously, $\hat{x} \in \mathcal{M}^{\prime}$.

For each $x$ in $\mathcal{M}$ and $f$ in $\mathcal{M}^{\prime}$, one can define a mapping $\theta_{x, f}$ from $\mathcal{M}$ into itself by follows: $\theta_{x, f} y=f(y) x$, for all $y \in \mathcal{M}$. Obviously, $\theta_{x, f} \in \operatorname{End}_{\mathcal{A}}(\mathcal{M})$.

In particular, for each $x, y$ in $\mathcal{M}$, we have $\theta_{x, \hat{y}} z=\hat{y}(z) x=\langle z, y\rangle x$, for all $z \in \mathcal{M}$.

For the operators of the above forms, we have the following lemmas.

Lemma 1.2. Let $\mathcal{M}$ be a Hilbert $C^{*}$-module over a $C^{*}$-algebra $\mathcal{A}$.

For all $a \in \mathcal{A}, x, y \in \mathcal{M}, f, g \in \mathcal{M}^{\prime}, A \in \operatorname{End}_{\mathcal{A}}(\mathcal{M})$, we have

(1) $\theta_{x, f} A=\theta_{x, f \circ A}$

(2) $A \theta_{x, f}=\theta_{A x, f}$

(3) if in addition, $\mathcal{A}$ is commutative, then $\theta_{x, f} \theta_{y, g}=f(y) \theta_{x, g}, \theta_{a x, f}=a \theta_{x, f}$.

Lemma 1.3. Let $\mathcal{M}$ be a Hilbert $C^{*}$-module over a $C^{*}$-algebra $\mathcal{A}$.

For all $a \in \mathcal{A}, x, y, z, w \in \mathcal{M}, A \in \operatorname{End}^{*}{ }_{\mathcal{A}}(\mathcal{M})$, we have 
(1) $\theta_{x, \hat{y}} \in \operatorname{End}_{\mathcal{A}}^{*}(\mathcal{M})$, and $\theta_{x, \hat{y}}^{*}=\theta_{y, \hat{x}}$,

(2) $\theta_{x, \hat{y}} A=\theta_{x, \hat{y} \circ A}=\theta_{x, \widehat{A^{*} y}}$,

(3) $A \theta_{x, \hat{y}}=\theta_{A x, \hat{y}}$

(4) if in addition, $\mathcal{A}$ is commutative, then $\theta_{x, \hat{y}} \theta_{z, \hat{w}}=\langle z, y\rangle \theta_{x, \hat{w}}, \theta_{a x, \hat{y}}=a \theta_{x, \hat{y}}=\theta_{x, \widehat{a^{*} y}}$.

For a commutative $\mathrm{C}^{*}$-algebra $\mathcal{A}$, for each $a$ in $\mathcal{A}$, one can define a mapping $T_{a}$ from $\mathcal{M}$ into itself by follows: $T_{a} x=a x$, for all $x \in \mathcal{M}$. Obviously, $T_{a} \in \operatorname{End}_{\mathcal{A}}(\mathcal{M})$. It is worthwhile to notice that if $\mathcal{A}$ is not commutative, then $T_{a}$ is not $\mathcal{A}$-linear. In this case, $T_{a}$ is not in $\operatorname{End}_{\mathcal{A}}(\mathcal{M})$.

Lemma 1.4. Let $\mathcal{A}$ be a commutative $C^{*}$-algebra with unit e and $\mathcal{M}$ be a full Hilbert $\mathcal{A}$ module. Then $\mathcal{Z}\left(\operatorname{End}_{\mathcal{A}}(\mathcal{M})\right)=\left\{T_{a}: a \in \mathcal{A}\right\}$.

Proof. For each $A$ in $\operatorname{End}_{\mathcal{A}}(\mathcal{M})$ and $x$ in $\mathcal{M}$, since $A T_{a} x=A(a x)=a A x=T_{a} A x$, we have $A T_{a}=T_{a} A$. It is to say $T_{a} \in \mathcal{Z}\left(\operatorname{End}_{\mathcal{A}}(\mathcal{M})\right)$.

On the other hand, assume $A \in \mathcal{Z}\left(\operatorname{End}_{\mathcal{A}}(\mathcal{M})\right)$. By Lemma 1.1, there exists a sequence $\left\{x_{i}\right\}_{i=1}^{n} \subseteq \mathcal{M}$, such that $\sum_{i=1}^{n}\left\langle x_{i}, x_{i}\right\rangle=e$. Thus we have

$$
\sum_{i=1}^{n} A \theta_{x, \hat{x}_{i}} x_{i}=\sum_{i=1}^{n}\left\langle x_{i}, x_{i}\right\rangle A x=A x
$$

and

$$
\sum_{i=1}^{n} \theta_{x, \hat{x_{i}}} A x_{i}=\sum_{i=1}^{n}\left\langle A x_{i}, x_{i}\right\rangle x .
$$

Let $\sum_{i=1}^{n}\left\langle A x_{i}, x_{i}\right\rangle=a$. Then we have $A=T_{a}$. The proof is complete.

For an algebra $\mathcal{A}$, if for each $a$ in $\mathcal{A}, a \mathcal{A} a=0$ implies that $a=0$, then it is said to be semi-prime.

Lemma 1.5. Let $\mathcal{A}$ be a $C^{*}$-algebra and $\mathcal{M}$ be a Hilbert $\mathcal{A}$-module. Then $\operatorname{End}_{\mathcal{A}}(\mathcal{M})$ is a semi-prime Banach algebra.

Proof. Let $A$ be in $\operatorname{End}_{\mathcal{A}}(\mathcal{M})$. Assume that $A B A=0$ for each $B$ in $\operatorname{End}_{\mathcal{A}}(\mathcal{M})$. In particular, for each $x \in \mathcal{M}$ and $f \in \mathcal{M}^{\prime}$, we have

$$
A \theta_{x, f} A x=\theta_{A x, f \circ A} x=f(A x) A x=0 .
$$

By taking $y=A x$ and $f=\hat{y}$, we have $\langle y, y\rangle y=0$. It follows that

$$
\langle\langle y, y\rangle y,\langle y, y\rangle y\rangle=\langle y, y\rangle^{3}=0 .
$$

Since $\langle y, y\rangle$ is a self-adjoint element, we have $\langle y, y\rangle=0$, and $y=0$. Hence $A=0$, and $\operatorname{End}_{\mathcal{A}}(\mathcal{M})$ is semi-prime. The proof is complete. 


\section{Derivations on $\operatorname{End}_{\mathcal{A}}(\mathcal{M})$}

In this section, we study derivations on $\operatorname{End}_{\mathcal{A}}(\mathcal{M})$. We begin with several lemmas.

Lemma 2.1. Let $\mathcal{A}$ be a commutative unital $C^{*}$-algebra and $\mathcal{M}$ be a full Hilbert $\mathcal{A}$ module. Then each derivation on $\operatorname{End}_{\mathcal{A}}(\mathcal{M})$ is $\mathcal{A}$-linear, i.e. $d(a A)=a d(A)$, for each $a \in \mathcal{A}$ and $A \in \operatorname{End}_{\mathcal{A}}(\mathcal{M})$.

Proof. Suppose $d$ is a derivation on $\operatorname{End}_{\mathcal{A}}(\mathcal{M})$.

By Lemma 1.4, we have $\mathcal{Z}\left(\operatorname{End}_{\mathcal{A}}(\mathcal{M})\right)=\left\{T_{a}: a \in \mathcal{A}\right\}$. For each $A$ in $\operatorname{End}_{\mathcal{A}}(\mathcal{M})$, By

$$
d\left(T_{a} A\right)=d\left(T_{a}\right) A+T_{a} d(A)
$$

and

$$
d\left(A T_{a}\right)=A d\left(T_{a}\right)+d(A) T_{a}
$$

we obtain $d\left(T_{a}\right) A=A d\left(T_{a}\right)$. Hence $d\left(T_{a}\right) \in \mathcal{Z}\left(\operatorname{End}_{\mathcal{A}}(\mathcal{M})\right)$, and $d\left(\mathcal{Z}\left(\operatorname{End}_{\mathcal{A}}(\mathcal{M})\right)\right) \subseteq$ $\mathcal{Z}\left(\operatorname{End}_{\mathcal{A}}(\mathcal{M})\right)$.

Since $\mathcal{Z}\left(\operatorname{End}_{\mathcal{A}}(\mathcal{M})\right)=\left\{T_{a}: a \in \mathcal{A}\right\}$ is a commutative $\mathrm{C}^{*}$-algebra, and every derivation on a commutative $\mathrm{C}^{*}$-algebra is zero, we have $d\left(T_{a}\right)=0$.

It follows that

$$
d(a A)=d\left(T_{a} A\right)=d\left(T_{a}\right) A+T_{a} d(A)=T_{a} d(A)=a d(A),
$$

which means that $d$ is $\mathcal{A}$-linear. The proof is complete.

Lemma 2.2. Let $\mathcal{A}$ be a commutative unital $C^{*}$-algebra and $\mathcal{M}$ be a full Hilbert $\mathcal{A}$ module. Then each derivation on $\operatorname{End}_{\mathcal{A}}(\mathcal{M})$ is continuous.

Proof. Suppose $d$ is a derivation on $\operatorname{End}_{\mathcal{A}}(\mathcal{M})$. Assume that $\left\{T_{n}\right\}$ is a sequence converging to zero in $\operatorname{End}_{\mathcal{A}}(\mathcal{M})$, and $\left\{d\left(T_{n}\right)\right\}$ converges to $T$.

According to the closed graph theorem, to show $d$ is continuous, it is sufficient to prove that $T=0$.

By Lemma 2.1, we know $d$ is $\mathcal{A}$-linear. For $x, y \in \mathcal{M}, f, g \in \mathcal{M}^{\prime}$, we have

$$
d\left(\theta_{x, f} T_{n} \theta_{y, g}\right)=d\left(f\left(T_{n} y\right) \theta_{x, g}\right)=f\left(T_{n} y\right) d\left(\theta_{x, g}\right) \rightarrow 0
$$

and

$$
d\left(\theta_{x, f} T_{n} \theta_{y, g}\right)=d\left(\theta_{x, f}\right) T_{n} \theta_{y, g}+\theta_{x, f} d\left(T_{n}\right) \theta_{y, g}+\theta_{x, f} T_{n} d\left(\theta_{y, g}\right) .
$$

Since $\left\{T_{n}\right\}$ converges to zero and $\left\{d\left(T_{n}\right)\right\}$ converges to $T$, we have

$$
d\left(\theta_{x, f} T_{n} \theta_{y, g}\right) \rightarrow \theta_{x, f} T \theta_{y, g}=f(T y) \theta_{x, g}
$$

It follows that $f(T y) \theta_{x, g}=0$. 
Let $a=f(T y)$, then we have $a \theta_{x, g}=\theta_{a x, g}=0$. For each $z \in \mathcal{M}$, we have

$$
\theta_{a x, g} z=g(z) a x=0 .
$$

By taking $g=\widehat{a x}$ and $z=a x$ in (2.1), we can obtain $a x=0$, i.e.

$$
f(T y) x=0 .
$$

By taking $f=\widehat{T y}$ and $x=T y$ in (2.2), we can obtain $T y=0$. i.e. $T=0$. The proof is complete.

Now we can prove our main theorem in this section.

Theorem 2.3. Let $\mathcal{A}$ be a commutative $C^{*}$-algebra with unit $e$ and $\mathcal{M}$ be a full Hilbert $\mathcal{A}$-module. Then each derivation on $\operatorname{End}_{\mathcal{A}}(\mathcal{M})$ is an inner derivation.

Proof. Suppose $d$ is a derivation on $\operatorname{End}_{\mathcal{A}}(\mathcal{M})$ and $\left\{x_{i}\right\}_{i=1}^{n}$ is a sequence in $\mathcal{M}$ such that $\sum_{i=1}^{n}\left\langle x_{i}, x_{i}\right\rangle=e$.

Define a mapping $T$ from $\mathcal{M}$ into itself by follows:

$$
T x=\sum_{i=1}^{n} d\left(\theta_{x, x_{i}}\right) x_{i}
$$

for all $x \in \mathcal{M}$.

By Lemmas 2.1 and 2.2, $d$ is $\mathcal{A}$-linear and continuous, thus $T$ is also $\mathcal{A}$-linear and continuous. That is to say $T \in \operatorname{End}_{\mathcal{A}}(\mathcal{M})$.

Now it is sufficient to show that $d(A)=T A-A T$, for each $A \in \operatorname{End}_{\mathcal{A}}(\mathcal{M})$.

For each $x \in \mathcal{M}$, we have

$$
\begin{aligned}
T A x & =\sum_{i=1}^{n} d\left(\theta_{A x, x_{i}}\right) x_{i} \\
& =\sum_{i=1}^{n} d\left(A \theta_{x, x_{i}}\right) x_{i} \\
& =\sum_{i=1}^{n} d(A) \theta_{x, x_{i}} x_{i}+\sum_{i=1}^{n} A d\left(\theta_{x, x_{i}}\right) x_{i} \\
& =d(A) \sum_{i=1}^{n}\left\langle x_{i}, x_{i}\right\rangle x+A \sum_{i=1}^{n} d\left(\theta_{x, x_{i}}\right) x_{i} \\
& =d(A) x+A T x .
\end{aligned}
$$

It implies that $d(A)=T A-A T$. Hence $d$ is an inner derivation. The proof is complete. 


\section{$3 \quad$ 2-Local derivations on $\operatorname{End}_{\mathcal{A}}(\mathcal{M})$ and $\operatorname{End}_{\mathcal{A}}^{*}(\mathcal{M})$}

In this section, we characterize 2-local derivations on $\operatorname{End}_{\mathcal{A}}(\mathcal{M})$ and $\operatorname{End}_{\mathcal{A}}^{*}(\mathcal{M})$. Firstly, we show the following lemma.

Lemma 3.1. Let $\mathcal{A}$ be a commutative unital $C^{*}$-algebra and $\mathcal{M}$ be a Hilbert $\mathcal{A}$-module. For $x_{i} \in \mathcal{M}$ and $f_{i} \in \mathcal{M}^{\prime}$, if $\sum_{i=1}^{n} \theta_{x_{i}, f_{i}}=0$, then $\sum_{i=1}^{n} f_{i}\left(x_{i}\right)=0$.

Proof. Let $a_{i, j}=f_{j}\left(x_{i}\right) \in \mathcal{A}$ and $\Lambda=\left(a_{i, j}\right)_{n \times n} \in M_{n}(\mathcal{A})$.

We have

$$
\begin{aligned}
& \sum_{i=1}^{n} f_{i}\left(x_{k}\right) x_{i}=\sum_{i=1}^{n} \theta_{x_{i}, f_{i}} x_{k}=0 \\
\Rightarrow & \sum_{i=1}^{n} f_{i}\left(x_{k}\right) f_{j}\left(x_{i}\right)=f_{j}\left(\sum_{i=1}^{n} f_{i}\left(x_{k}\right) x_{i}\right)=0 \\
\Rightarrow & \sum_{i=1}^{n} a_{k, i} a_{i, j}=0 \\
\Rightarrow & \Lambda^{2}=0 .
\end{aligned}
$$

Since $\mathcal{A}$ is a commutative unital $\mathrm{C}^{*}$-algebra, it is well known that $\mathcal{A}$ is $*$-isomorphic to $C(\mathcal{S})$ for some compact Hausdorff space $\mathcal{S}$. Without loss of generality, we can assume $\mathcal{A}=C(\mathcal{S})$.

Then for each $t \in \mathcal{S}$, we have $a_{i, j}(t) \in \mathbb{C}$ and $\Lambda(t), \Lambda^{2}(t) \in M_{n}(\mathbb{C})$.

Recall that for a matrix $A$ in $M_{n}(\mathbb{C}), A^{2}=0$ implies that $\operatorname{tr}(A)=0$, where $\operatorname{tr}(A)$ denotes the trace of $A$, i.e. the sum of all the diagonal elements.

Hence $\Lambda^{2}(t)=0$ implies that $\operatorname{tr}(\Lambda(t))=0$. It follows that $\operatorname{tr}(\Lambda)=0$, that is to say $\sum_{i=1}^{n} f_{i}\left(x_{i}\right)=0$. The proof is complete.

Theorem 3.2. Let $\mathcal{A}$ be a commutative unital $C^{*}$-algebra and $\mathcal{M}$ be a full Hilbert $\mathcal{A}$ module. Then each 2-local derivation on $\operatorname{End}_{\mathcal{A}}(\mathcal{M})$ is a derivation.

Proof. Denote by $\Gamma(\mathcal{M})$ the linear span of the set $\left\{\theta_{x, f}: x \in \mathcal{M}, f \in \mathcal{M}^{\prime}\right\}$. By Lemma 1.2. $\Gamma(\mathcal{M})$ is a two-side ideal of $\operatorname{End}_{\mathcal{A}}(\mathcal{M})$.

For each $S=\sum_{i=1}^{n} \theta_{x_{i}, f_{i}} \in \Gamma(\mathcal{M})$, define $\phi(S)=\sum_{i=1}^{n} f_{i}\left(x_{i}\right)$.

One can verify that $\phi$ is well defined by Lemma 3.1. And obviously, $\phi$ is $\mathcal{A}$-linear. Moreover, for each $A \in \operatorname{End}_{\mathcal{A}}(\mathcal{M})$, we have

$$
\phi\left(\theta_{x, f} A\right)=\phi\left(\theta_{x, f \circ A}\right)=f(A x)=\phi\left(\theta_{A x, f}\right)=\phi\left(A \theta_{x, f}\right) .
$$

It follows that $\phi(S A)=\phi(A S)$ for each $A \in \operatorname{End}_{\mathcal{A}}(\mathcal{M})$ and $S \in \Gamma(\mathcal{M})$.

Suppose $\delta$ is a 2-local derivation on $\operatorname{End}_{\mathcal{A}}(\mathcal{M})$. By the definition of 2-local derivation, there exists a derivation $d$ on $\operatorname{End}_{\mathcal{A}}(\mathcal{M})$ such that $\delta(A)=d(A)$ and $\delta(S)=d(S)$. 
By Theorem 2.3, $d$ is an inner derivation, i.e. there exists an element $T \in \operatorname{End}_{\mathcal{A}}(\mathcal{M})$ such that $d=D_{T}$.

Thus we have

$$
\delta(A) S+A \delta(S)=d(A) S+A d(S)=d(A S)=D_{T}(A S)=T A S-A S T .
$$

Since $\Gamma(\mathcal{M})$ is a two-side ideal of $\operatorname{End}_{\mathcal{A}}(\mathcal{M})$, we know that $A S \in \Gamma(\mathcal{M})$.

Hence

$$
\phi(\delta(A) S+A \delta(S))=\phi(T A S-A S T)=0,
$$

which follows that $\phi(\delta(A) S)=-\phi(A \delta(S))$.

Now, for each $A, B \in \operatorname{End}_{\mathcal{A}}(\mathcal{M})$ and $S \in \Gamma(\mathcal{M})$, we have

$$
\begin{aligned}
\phi(\delta(A+B) S) & =-\phi((A+B) \delta(S)) \\
& =-\phi(A \delta(S))-\phi(B \delta(S)) \\
& =\phi(\delta(A) S)+\phi(\delta(B) S) \\
& =\phi((\delta(A)+\delta(B)) S) .
\end{aligned}
$$

Let $C=\delta(A+B)-\delta(A)-\delta(B)$, we obtain $\phi(C S)=0$.

By taking $S=\theta_{x, f}$, we have

$$
\phi\left(C \theta_{x, f}\right)=f(C x)=0 \Rightarrow\langle C x, C x\rangle=0 \Rightarrow C x=0 \Rightarrow C=0 .
$$

It means that $\delta(A+B)=\delta(A)+\delta(B)$. That is to say $\delta$ is an additive mapping. In addition, by the definition of 2-local derivation, it is easy to show that $\delta$ is homogeneous and $\delta\left(A^{2}\right)=A \delta(A)+\delta(A) A$ for each $A \in \operatorname{End}_{\mathcal{A}}(\mathcal{M})$. Hence $\delta$ is a Jordan derivation.

By Lemma 1.5. $\operatorname{End}_{\mathcal{A}}(\mathcal{M})$ is a semi-prime Banach algebra. According to the classical result that every Jordan derivation on a semi-prime Banach algebra is a derivation [5], we obtain that $\delta$ is a derivation. The proof is complete.

Theorem 3.3. Let $\mathcal{A}$ be a commutative unital $C^{*}$-algebra and $\mathcal{M}$ be a full Hilbert $\mathcal{A}$ module. Then each 2-local derivation on $\operatorname{End}_{\mathcal{A}}(\mathcal{M})$ is a derivation.

Proof. Denote by $\Gamma^{*}(\mathcal{M})$ the linear span of the set $\left\{\theta_{x, \hat{y}}: x, y \in \mathcal{M}\right\}$. By Lemma 1.3. $\Gamma^{*}(\mathcal{M})$ is a two-side ideal of $\operatorname{End}_{\mathcal{A}}^{*}(\mathcal{M})$.

For each $S=\sum_{i=1}^{n} \theta_{x_{i}, \widehat{y}_{i}} \in \Gamma^{*}(\mathcal{M})$, define $\phi(S)=\sum_{i=1}^{n}\left\langle x_{i}, y_{i}\right\rangle$.

By Lemma 3.1, $\phi$ is well defined. For each $A \in \operatorname{End}_{\mathcal{A}}^{*}(\mathcal{M})$, we have

$$
\phi\left(\theta_{x, \widehat{y}} A\right)=\phi\left(\theta_{x, \widehat{A^{*} y}}\right)=\left\langle x, A^{*} y\right\rangle=\langle A x, y\rangle=\phi\left(\theta_{A x, \widehat{y}}\right)=\phi\left(A \theta_{x, \widehat{y}}\right) .
$$

It follows that $\phi(S A)=\phi(A S)$ for each $A \in \operatorname{End}_{\mathcal{A}}^{*}(\mathcal{M})$ and $S \in \Gamma^{*}(\mathcal{M})$.

In [15], the authors prove that for a commutative unital $\mathrm{C}^{*}$-algebra $\mathcal{A}$ and a full Hilbert $\mathcal{A}$-module $\mathcal{M}$, each derivation on $\operatorname{End}_{\mathcal{A}}^{*}(\mathcal{M})$ is an inner derivation.

The rest of the proof is similar to Theorem 3.2 , so we omit it. 


\section{Local derivations on $\operatorname{End}_{\mathcal{A}}(\mathcal{M})$}

In this section, we discuss local derivations on $\operatorname{End}_{\mathcal{A}}(\mathcal{M})$. Through this section, we assume that $\mathcal{A}$ is a commutative $\mathrm{C}^{*}$-algebra with unit $e$, and $\mathcal{M}$ is a Hilbert $\mathcal{A}$-module, and moreover, there exist $x_{0}$ in $\mathcal{M}$ and $f_{0}$ in $\mathcal{M}^{\prime}$ such that $f_{0}\left(x_{0}\right)=e$. Denote the unit of $\operatorname{End}_{\mathcal{A}}(\mathcal{M})$ by $I$. Define $\mathcal{L}=\operatorname{span}\left\{\theta_{x, f_{0}}: x \in \mathcal{M}\right\}$, and $\mathcal{R}=\operatorname{span}\left\{\theta_{x_{0}, f}: f \in \mathcal{M}^{\prime}\right\}$.

\section{Lemma 4.1.}

(1) $\theta_{x_{0}, f_{0}}$ is an idempotent;

(2) each element in $\mathcal{L}$ is an $\mathcal{A}$-linear combination of some idempotents in $\mathcal{L}$, and each element in $\mathcal{R}$ is an $\mathcal{A}$-linear combination of some idempotents in $\mathcal{R}$;

(3) $\mathcal{L}$ is a left ideal of $\operatorname{End}_{\mathcal{A}}(\mathcal{M})$, and $\mathcal{R}$ is a right ideal of $\operatorname{End}_{\mathcal{A}}(\mathcal{M})$;

(4) $\mathcal{L}$ is a left separating set of $\operatorname{End}_{\mathcal{A}}(\mathcal{M})$, i.e. for each $A$ in $\operatorname{End}_{\mathcal{A}}(\mathcal{M}), A \mathcal{L}=0$ implies that $A=0$, and $\mathcal{R}$ is a right separating set of $\operatorname{End}_{\mathcal{A}}(\mathcal{M})$, i.e. for each $A$ in $\operatorname{End}_{\mathcal{A}}(\mathcal{M})$, $\mathcal{R} A=0$ implies that $A=0$

Proof. (1) $\theta_{x_{0}, f_{0}} \theta_{x_{0}, f_{0}}=f_{0}\left(x_{0}\right) \theta_{x_{0}, f_{0}}=\theta_{x_{0}, f_{0}}$.

(2) For each $x \in \mathcal{M}$, there exists a non-zero complex number $\lambda \in \mathbb{C}$, such that $e-\lambda f_{0}(x)$ is invertible in $\mathcal{A}$. Denote $e-\lambda f_{0}(x)$ by $a^{-1}$, then we have

$$
f_{0}\left(a\left(x_{0}-\lambda x\right)\right)=a f_{0}\left(x_{0}-\lambda x\right)=a\left(e-\lambda f_{0}(x)\right)=a a^{-1}=e .
$$

By (1), we know that $\theta_{a\left(x_{0}-\lambda x\right), f_{0}}$ is an idempotent.

Thus we have

$$
\theta_{x, f_{0}}=\lambda^{-1} \theta_{x_{0}, f_{0}}-\lambda^{-1} a^{-1} \theta_{a\left(x_{0}-\lambda x\right), f_{0}} .
$$

That is to say $\theta_{x, f_{0}}$ is an $\mathcal{A}$-linear combination of idempotents in $\mathcal{L}$.

Similarly, for each $f \in \mathcal{M}^{\prime}$, there exists a non-zero complex number $\lambda \in \mathbb{C}$, such that $e-\lambda f\left(x_{0}\right)$ is invertible in $\mathcal{A}$. Denote $e-\lambda f\left(x_{0}\right)$ by $a^{-1}$, then we have

$$
\left(a\left(f_{0}-\lambda f\right)\right)\left(x_{0}\right)=a\left(e-\lambda f\left(x_{0}\right)\right)=a a^{-1}=e .
$$

Again by (1), we know that $\theta_{x_{0}, a\left(f_{0}-\lambda f\right)}$ is an idempotent.

Thus we have

$$
\theta_{x_{0}, f}=\lambda^{-1} \theta_{x_{0}, f_{0}}-\lambda^{-1} a^{-1} \theta_{x_{0}, a\left(f_{0}-\lambda f\right)} .
$$

(3) For each $A \in \operatorname{End}_{\mathcal{A}}(\mathcal{M})$, since $A \theta_{x, f_{0}}=\theta_{A x, f_{0}}$, we know that $\mathcal{L}$ is a left ideal of $\operatorname{End}_{\mathcal{A}}(\mathcal{M})$. Similarly, $\mathcal{R}$ is a right ideal of $\operatorname{End}_{\mathcal{A}}(\mathcal{M})$ since $\theta_{x_{0}, f} A=\theta_{x_{0}, f \circ A}$.

(4) Suppose $A \in \operatorname{End}_{\mathcal{A}}(\mathcal{M}), \theta_{x, f_{0}} \in \mathcal{L}, \theta_{x_{0}, f} \in \mathcal{R}$.

If $A \theta_{x, f_{0}}=0$, then

$$
0=A \theta_{x, f_{0}} x_{0}=\theta_{A x, f_{0}} x_{0}=f_{0}\left(x_{0}\right) A x=A x,
$$


i.e. $A=0$.

If $\theta_{x_{0}, f} A=0$, then

$$
\begin{aligned}
& \theta_{x_{0}, f} A x=f(A x) x_{0}=0 \\
\Rightarrow & f_{0}\left(f(A x) x_{0}\right)=f(A x) f_{0}\left(x_{0}\right)=f(A x)=0 \\
\Rightarrow & \langle A x, A x\rangle=0 \\
\Rightarrow & A x=0 \\
\Rightarrow & A=0 .
\end{aligned}
$$

The proof is complete.

Let $\mathcal{J}$ be a left $\mathcal{A}$-module, and $\phi$ be a bilinear mapping from $\operatorname{End}_{\mathcal{A}}(\mathcal{M}) \times \operatorname{End}_{\mathcal{A}}(\mathcal{M})$ into $\mathcal{J}$.

We say that $\phi$ is $\mathcal{A}$-bilinear if $\phi(a A, B)=\phi(A, a B)=a \phi(A, B)$ for each $A, B \in$ $\operatorname{End}_{\mathcal{A}}(\mathcal{M})$ and $a \in \mathcal{A}$.

We say that $\phi$ preserves zero product if $A B=0$ implies that $\phi(A, B)=0$ for each $A, B \in \operatorname{End}_{\mathcal{A}}(\mathcal{M})$.

Lemma 4.2. Let $\mathcal{J}$ be a left $\mathcal{A}$-module, and $\phi: \operatorname{End}_{\mathcal{A}}(\mathcal{M}) \times \operatorname{End}_{\mathcal{A}}(\mathcal{M}) \rightarrow \mathcal{J}$ be an $\mathcal{A}$-bilinear mapping preserving zero product. Then for each $A, B \in \operatorname{End}_{\mathcal{A}}(\mathcal{M}), L \in \mathcal{L}$, and $R \in \mathcal{R}$, we have:

$$
\phi(A, L B)=\phi(A L, B)=\phi(I, A L B)
$$

and

$$
\phi(A R, B)=\phi(A, R B)=\phi(A R B, I) .
$$

Proof. Suppose $P$ is an idempotent in $\operatorname{End}_{\mathcal{A}}(\mathcal{M})$. Let $Q=I-P$.

Since $\phi$ preserves zero product, we have

$$
\phi(A, P B)=\phi(A P+A Q, P B)=\phi(A P, P B)=\phi(A P, B-Q B)=\phi(A P, B) .
$$

By Lemma 4.1(2), each element in $\mathcal{L}$ is an $\mathcal{A}$-linear combination of idempotents in $\mathcal{L}$. Considering $\phi$ is $\mathcal{A}$-bilinear, we obtain that $\phi(A, L B)=\phi(A L, B)$.

By Lemma $4.1(3), \mathcal{L}$ is a left ideal, so $A L \in \mathcal{L}$. Hence $\phi(A L, B)=\phi(I, A L B)$.

Similarly, we can show the equation (4.2) is true.

For an algebra $\mathcal{A}$ with unit $e$, a linear mapping $\delta$ on $\mathcal{A}$ is said to be a generalized derivation if $\delta(a b)=a \delta(b)+\delta(a) b-a \delta(e) b$, for all $a, b$ in $\mathcal{A}$.

Theorem 4.3. Suppose that $\mathcal{A}$ is a commutative $C^{*}$-algebra with unit e, and $\mathcal{M}$ is a Hilbert $\mathcal{A}$-module, and moreover, there exist $x_{0}$ in $\mathcal{M}$ and $f_{0}$ in $\mathcal{M}^{\prime}$ such that $f_{0}\left(x_{0}\right)=e$. If $\delta$ is an $\mathcal{A}$-linear mapping from $\operatorname{End}_{\mathcal{A}}(\mathcal{M})$ into itself such that: for each $A, B, C$ in $\operatorname{End}_{\mathcal{A}}(\mathcal{M}), A B=B C=0$ implies that $A \delta(B) C=0$, then $\delta$ is a generalized derivation. In particular, if $\delta(I)=0$, where $I$ is the unit of $\operatorname{End}_{\mathcal{A}}(\mathcal{M})$, then $\delta$ is a derivation. 
Proof. Suppose $A, B, X, Y, A_{0}, B_{0}$ are arbitrary elements in $\operatorname{End}_{\mathcal{A}}(\mathcal{M})$, where $A_{0} B_{0}=$ $0, L$ and $R$ are arbitrary elements in $\mathcal{L}$ and $\mathcal{R}$, respectively.

Define a bilinear mapping $\phi_{1}: \phi_{1}(X, Y)=X \delta\left(Y A_{0}\right) B_{0}$. Then $\phi_{1}$ is an $\mathcal{A}$-bilinear mapping preserving zero product.

By Lemma 4.2, we have

$$
\phi_{1}(R, A)=\phi_{1}(R A, I)
$$

i.e.

$$
R \delta\left(A A_{0}\right) B_{0}=R A \delta\left(A_{0}\right) B_{0}
$$

Since $\mathcal{R}$ is a right separating set of $\operatorname{End}_{\mathcal{A}}(\mathcal{M})$, we have

$$
\delta\left(A A_{0}\right) B_{0}=A \delta\left(A_{0}\right) B_{0} .
$$

Now define a bilinear mapping $\phi_{2}: \phi_{2}(X, Y)=\delta(A X) Y-A \delta(X) Y$. Then $\phi_{2}$ is also an $\mathcal{A}$-bilinear mapping preserving zero product.

Again by Lemma 4.2, we have

$$
\phi_{2}(B, L)=\phi_{2}(I, B L)
$$

i.e.

$$
\delta(A B) L-A \delta(B) L=\delta(A) B L-A \delta(I) B L .
$$

Since $\mathcal{L}$ is a left separating set of $\operatorname{End}_{\mathcal{A}}(\mathcal{M})$, we obtain that

$$
\delta(A B)=A \delta(B)+\delta(A) B-A \delta(I) B .
$$

That is to say $\delta$ is a generalized derivation. The proof is complete.

Applying the above Theorem, we can get the following corollary immediately.

Corollary 4.4. Suppose $\mathcal{A}$ is a commutative $C^{*}$-algebra with unit e, $\mathcal{M}$ is a Hilbert $\mathcal{A}$ module, and moreover, there exist $x_{0}$ in $\mathcal{M}$ and $f_{0}$ in $\mathcal{M}^{\prime}$ such that $f_{0}\left(x_{0}\right)=e$. Then each $\mathcal{A}$-linear local derivation $\delta$ on $\operatorname{End}_{\mathcal{A}}(\mathcal{M})$ is a derivation.

Proof. For each $A, B, C$ in $\operatorname{End}_{\mathcal{A}}(\mathcal{M})$, if $A B=B C=0$, by the definition of local derivation, there exists a derivation $\delta_{B}$ such that $\delta_{B}(B)=\delta(B)$. Thus we have

$$
A \delta(B) C=A \delta_{B}(B) C=\delta_{B}(A B C)-\delta_{B}(A) B C-A B \delta_{B}(C)=0
$$

Let $I$ be the unit of $\operatorname{End}_{\mathcal{A}}(\mathcal{M})$, by the definition of local derivation, there exists a derivation $\delta_{I}$ such that $\delta_{I}(I)=\delta(I)=0$.

By Theorem 4.3, $\delta$ is a derivation. The proof is complete.

Acknowledgements. This paper was partially supported by National Natural Science Foundation of China(Grant No. 11371136). 


\section{References}

[1] S. Ayupov, K. Kudaybergenov, 2-local derivations on von Neumann algebras, Positivity, 19(2014), 445-455.

[2] S. Ayupov, K. Kudaybergenov, A. Peralta, A survey on local and 2-local derivations on $\mathrm{C}^{*}$ - and von Neuman algebras, Topics in functional analysis and algebra, 73-126, Contemp Math., 672, 2016.

[3] E. Christensen, Derivations of nest algebras, Math. Ann., 229(1977), 155-161.

[4] R. Crist, Local derivations on operator algebras, J. Funct. Anal., 135(1996), 72-92.

[5] J. Cusack, Jordan derivations on rings, Proc. Amer. Math. Soc., 53(1975), 321-324.

[6] D. Hadwin, J. Li, Local derivations and local automorphisms on some algebras, J. Operator Theory, 60(2008), 29-44.

[7] J. He, J. Li, G. An, W. Huang, Characterizations of 2-local derivations and local Lie derivations on some algebras, Sib. Math. J., to appear.

[8] B. Johnson, Local derivations on $\mathrm{C}^{*}$-algebras are derivations, Trans. Amer. Math. Soc., 353(2001), 313-325.

[9] R. Kadison, Derivations of operator algebras, Ann. Math., 83(1966), 280-293.

[10] R. Kadison, Local derivations, J. Algebra, 130(1990), 494-509.

[11] S. Kim, J. Kim, Local automorphisms and derivations on $M_{n}(\mathbb{C})$, Proc. Amer. Math. Soc., 132(2004), 1389-1392.

[12] E. Lance, Hilbert C*-Modules: A Toolkit for Operator Algebraists, Cambridge University Press, 1995.

[13] D. Larson, A. Sourour, Local derivations and local automorphisms, Proc. Sympos. Pure Math., 51(1990), 187-194.

[14] J. Li, Z. Pan, Annihilator-preserving maps, multipliers and local derivations, Linear Algebra Appl., 432(2010), 5-13.

[15] P. Li, D. Han, W. Tang, Derivations on the algebra of operators in Hilbert C*modules, Acta Math. Sinica(Engl. Ser.), 28(2012), 1615-1622.

[16] M. Moghadam, M. Miri, A. Janfada, A note on derivations on the algebra of operators in Hilbert C*-modules, Mediterr. J. Math., 13(2016), 1167-1175.

[17] S. Sakai, Derivations of $W^{*}$-algebras, Ann. Math., 83(1966), 273-279.

[18] P. Šemrl, Local automorphisms and derivations on $B(H)$, Proc. Amer. Math. Soc., 125(1997), 2677-2680.

[19] J. Zhang, H. Li, 2-Loacl derivations on digraph algebras, Acta Math. Sinica(Chin. Ser.), 49(2006), 1401-1406. 